\title{
Mechanisms of arm paresis in middle cerebral artery distribution stroke: Pilot study
}

\author{
Vishnumurthy S. Hedna, MD; ${ }^{1}$ Sandip Jain, MD; ${ }^{2}$ Omid Rabbani, MD; ${ }^{2}$ Stephen E. Nadeau, MD $^{1,3^{*}}$ \\ ${ }^{1}$ Department of Neurology, University of Florida, Gainesville, FL; ${ }^{2}$ Neurology private practice; ${ }^{3}$ Neurology Service and \\ Brain Rehabilitation Research Center, Malcom Randall Department of Veterans Affairs Medical Center, Gainesville, FL
}

\begin{abstract}
The development of effective neurobiological adjuvants to rehabilitation of paresis caused by stroke will depend on understanding mechanisms of paresis. Our objective was to determine the extent to which upper-limb (UL) paresis after nonlacunar ischemic middle cerebral artery (MCA) distribution stroke is caused by infarction of posterior periventricular white matter (PVWM), where corticospinal fibers serving movement descend, and caused by infarction of the arm-hand region of precentral gyrus (ahPCG). We conducted a blinded, retrospective analysis of computed tomography and magnetic resonance imaging from a convenience sample of 64 prospectively evaluated subjects with UL paresis resulting from MCA distribution stroke. Of the subjects, 96.5\% had PVWM involvement while $53 \%$ had minimal or no ahPCG involvement. Even in subjects with no UL function, $56 \%$ had very minimal infarction $(<25 \%)$ of the ahPCG. Degree of paresis was statistically associated with presence or absence of detectable posterior PVWM damage but not with extent of ahPCG involvement. These preliminary findings suggest that posterior PVWM involvement may be a major, if not the principal, determinant of paresis in all hemispheric ischemic strokes and motivate further prospective studies of this problem.
\end{abstract}

Key words: arm paresis, corticospinal fibers, ischemic stroke, mechanisms of paresis, middle cerebral artery, motor cortex, neurobiological adjuvants, paresis, periventricular white matter, stroke, white matter.

\section{INTRODUCTION}

It is well established that the primary cause of hemiparesis following hemispheric stroke is damage to the motor cortex or the white matter pathways in the centrum semiovale, periventricular white matter (PVWM), or the posterior limb of the internal capsule linking this cortex to the brain stem and spinal cord [1-10]. The centrum semiovale and posterior PVWM contain the corona radiata, which includes fibers originating in the motor cortex that continue ventrally to comprise the posterior limb of the internal capsule. Damage to other areas that project to the brain stem or interact with motor cortex, including

Abbreviations: $\mathrm{AC}=$ anterior commissure, $\mathrm{ACA}=$ anterior cerebral artery, ahPCG $=$ arm-hand region of precentral gyrus, $\mathrm{BRRC}=$ Brain Rehabilitation Research Center, CIMT $=$ constraint-induced movement therapy, $\mathrm{CT}=$ computed tomography, DTT = diffusion tensor tractographic, $\mathrm{fMRI}=$ functional magnetic resonance imaging, MAL $=$ Motor Activity Log, MCA $=$ middle cerebral artery, MRI $=$ magnetic resonance imaging, $\mathrm{PC}=$ posterior commissure, $\mathrm{PVWM}=$ periventricular white matter, $\mathrm{SD}=$ standard deviation, $\mathrm{UL}=$ upper limb, $\mathrm{VA}=$ Department of Veterans Affairs.

*Address all correspondence to Stephen E. Nadeau, MD; Research Service (151), Malcom Randall VA Medical Center, 1601 SW Archer Rd, Gainesville, FL 32608; 352-3746082; fax: 352-374-6153. Email snadeau@ufl.edu http://dx.doi.org/10.1682/JRRD.2012.10.0194 
motor neurons in the postcentral gyrus, the premotor cortex, the supplementary motor area, and the cingulate motor area, or to their white matter projections may contribute to paresis, but in ways as yet not well defined. Damage to ancillary structures such as the basal ganglia and their projections may also contribute to paresis, again in uncertain ways.

With lacunar infarctions, both upper-limb (UL) and lower-limb paresis are related purely to a white matter lesion [11]. Lower-limb paresis in all hemispheric strokes is related to a white matter lesion except in the case of anterior cerebral artery (ACA) territory infarctions, which are uncommon (e.g., 10 of 408 in Duncan et al. [12]). However, the situation is not so straightforward in the case of middle cerebral artery (MCA) territory stroke. Clearly, lower-limb paresis in such strokes is related to a white matter lesion because portions of the motor cortex supporting lower-limb movement are spared. However, UL paresis might be primarily related to a cortical lesion, involving at the least the arm-hand region of the motor cortex - the conventional wisdom — or it might be related to a subcortical white matter lesion. Some studies have demonstrated a high prevalence of white matter lesions in the PVWM and centrum semiovale with nonlacunar MCA territory infarcts [13] and that motor deficits may be as severe with infarcts limited to the deep white matter as with large MCA territorial infarcts [14]. However, no study to our knowledge has addressed the specific question of relative contribution of cortical and white matter damage to UL paresis in MCA ischemic stroke. In our clinical observation, UL paresis associated with MCA territory infarcts has also seemed to usually be caused by damage to white matter pathways, most often in the posterior PVWM or the adjacent centrum semiovale. In this pilot study, which couples a prospective classification of motor function with a retrospective analysis of imaging studies using very simple methodology, we sought to determine whether there is enough evidence favoring our white matter lesion observation to motivate further studies employing more sophisticated methodology.

The primary mechanism of paresis in hemispheric stroke is of critical importance to the development of effective neurobiological strategies to rehabilitate paresis, which are urgently needed. Phase III stroke rehabilitation trials [12,15-16], while they have demonstrated the efficacy of particular rehabilitation strategies and their effect on long-term outcome, have also shown that, despite the intensity and dose of the treatments used, effect was modest, few patients recovered close to normal function, and many patients were left with significant impairment. Long-term impairment is even greater in patients who did not have sufficient baseline motor function to qualify for these trials. Although it can be anticipated that future refinements in experience-dependent neurorehabilitation will achieve better outcomes, it seems highly unlikely that any experience-dependent therapy, however effective, will provide a fully adequate rehabilitation approach to the full spectrum of motoric deficits seen after stroke. Enhancement of the substrate for experience-dependent neuroplasticity seems essential. But which substrate: gray matter or white matter? If it is established that white matter lesions are the predominant cause of paresis in most hemispheric strokes, then neurobiological strategies aimed at mitigating axonal damage and promoting axonal regrowth will be the greatest priority, as will the development of animal models to advance such strategies. It also seems likely that, as challenging as the development of neurobiological interventions for white matter damage is likely to be, it may be more readily feasible than regenerating, connecting, and retraining cerebral cortex.

\section{METHODS}

\section{Subjects}

This study involved a blinded, retrospective analysis of computed tomography (CT) and magnetic resonance imaging (MRI) conducted on a convenience sample of 64 subjects with chronic UL paresis resulting exclusively from MCA distribution infarction who were recruited through the Department of Veterans Affairs (VA) Rehabilitation Research and Development Service-funded Brain Rehabilitation Research Center (BRRC) and physically examined prospectively pursuant to their participation in rehabilitation research protocols. The BRRC recruitment and database programs have been approved by the University of Florida Institutional Review Board and all subjects provided informed consent to participate.

Inclusion criteria included (1) a single large vessel distribution infarction that was located in the MCA territory; (2) the availability of brain imaging studies, usually obtained at community hospitals or the VA hospital as part of the diagnostic evaluation during the original stroke hospitalization, that were of sufficient quality to provide the basis for the analyses involved in this investi- 
gation; and (3) the availability of a well-documented onsite evaluation of UL function at our research center that would provide the basis for the functional classification employed in this investigation. Subjects with a history of more than one stroke and subjects with lacunar infarcts were excluded. Lacunar infarcts were defined as small subcortical infarcts visible on two or more scan cuts, typically less then $15 \mathrm{~mm}$ in axial diameter, tracking a single penetrating vessel (usually a lenticulostriate artery) along its ventro-dorsal path, with maximum extent typically in the PVWM [11].

\section{Procedure}

The arm-hand region of precentral gyrus (ahPCG) was mapped without knowledge of severity of paresis on Matsui and Hirano atlas templates [17] (supplemented by Damasio atlas templates [18]). Fluid-attenuated inversion recovery MRI sequences were used when available, but when not, T2-weighted MRI sequences or CTs were used. Diffusion-weighted images were not available during the period of data collection. The atlases were also used to calculate the angle of the scan for each subject.

The ahPCG was defined according to the landmarks established by the functional MRI (fMRI) study of Crafton et al. [19] as the region extending dorsally from a line drawn parallel to the Sylvian fissure from the top of pars opercularis to a line drawn through the superior frontal sulcus (see also Meier et al. [20]). The anterior and posterior margins of the arm-hand region were defined by the precentral and central sulci, respectively. The entire region was accepted as being homogeneous because microstimulation mapping studies in squirrel monkeys [21] and fMRI studies in human subjects [19-20,22] have indicated that this region is a representational mosaic that does not have a somatotopic representational pattern. Calculation of the percent of arm-hand region involved for each subject was done by measuring the extent of ahPCG involvement on each slice and averaging over all slices that included the arm-hand region. Attempts were not made to map the extent of lesions of premotor cortex because we felt that such assessment would not be sufficiently reliable given the intrinsic limitations of our methods.

The subcortical white matter extent of lesions was defined on the slice from the Matsui and Hirano atlas of maximal white matter involvement [17]. Using Photoshop (Adobe Systems Inc; San Jose, California), the entire lesion in that slice was outlined manually and cop- ied onto the appropriate slice in the template slice series corresponding to the orbitomeatal line, i.e., the $0^{\circ}$ template (the orbitomeatal line is approximately $9^{\circ}$ steeper than the Talairach anterior commissure [AC]-posterior commissure [PC] line [23]). To correct for the effects of different scan angles, the lesion was then moved backward or forward on this template using the formula (Equation (1)):

$$
\text { Displacement }=100 \mathrm{X} / \mathrm{Y} \tan \theta=39.3 \tan \theta,
$$

where displacement was the percentage of the anteriorposterior dimension of the slice that the lesion was moved, and $\theta$ was the angle at which the scan was obtained relative to the orbitomeatal line. $\mathrm{X}$ is the distance between the external auditory meatus and the midbody of the lateral ventricle. $\mathrm{Y}$ is the average anteriorposterior dimension of the brain at the midventricular level. X and Y were measured from sagittal T1-weighted MRIs of 10 nondisabled adult men and 10 nondisabled adult women. The $\mathrm{X} / \mathrm{Y}$ ratio was $0.397 \pm 0.038$ (mean \pm standard deviation [SD]) for the men and $0.388 \pm 0.042$ for the women. These were not significantly different $(t=$ $0.503, p=0.62)$. Therefore, we used the mean of these two values: 0.393 . In most cases (especially with CT), the difference between the actual angle of the scan relative to the orbitomeatal line and $0^{\circ}$ was a positive number and the effect was to move the lesion posteriorly. However, if the scan was obtained at a negative angle (as in some MRIs), remapping to $0^{\circ}$ had the effect of moving the lesion anteriorly. Posterior PVWM was operationally defined as the posterior half of the white matter extending along the body of the lateral ventricle.

In order to determine whether cortical involvement played a greater role in patients with more severe paresis, degree of UL paresis was classified by the senior investigator (S.N.). Our classification criteria were originally developed for their heuristic value in allocating subjects to rehabilitation protocols. We employed this simple classification scheme simply to assure that our findings would generalize to all degrees of paresis. The classification scheme was not difficult to apply because it reflects what the BRRC does on a daily basis in determining subject treatment protocol eligibility. Because of the limited purpose of this classification scheme as used here (to assure a full spectrum of paresis), a modest number of misclassifications would not have altered the results and conclusions. Degree of paresis, determined at the time of 
subject screening, was not revealed until lesions had been mapped. The classification scheme is from 1 to 5 , as follows:

1. Normal examination.

2. Mild impairment ranging from pronation drift to slowing of movement, distal weakness (especially of extensors), reduction of capacity for individuated finger movement, and a Motor Activity Log (MAL) [16] quantitative score of $\geq 3$.

3. Moderate impairment seriously interfering with use of the UL (MAL quantitative score of $<3$ ) but sufficient distal extensor function to enable enough finger and thumb extension to enable some grip. Criteria met for constraint-induced movement therapy (CIMT).

4. Severe impairment. Some distal movement, e.g., grip, but insufficient wrist and finger extension to enable even slight opening of the hand, hence inability to meet CIMT criteria and no use of the UL in daily life.

5. No voluntary movement of UL, proximal or distal.

\section{Statistical Analysis}

The Fisher exact test was used to test the relationship between presence or absence of posterior PVWM involvement and the presence of some UL functionality (defined as class 1, 2, or 3 UL impairment). A Spearman correlation was used to test the relationship between severity of paresis and extent of involvement of ahPCG.

\section{RESULTS}

Mean \pm SD age of the subjects was $59 \pm 12$ yr (range: 25-81 yr). There were 44 men. Scans used were obtained $<1$ mo poststroke in 41 subjects: delay $4.1 \pm 5.5 \mathrm{~d}$ (range: 1-23 d). Scans were obtained $>1$ mo poststroke in 23 subjects: delay $4.0 \pm 6.9 \mathrm{yr}$ (range: $0.1-31.0 \mathrm{yr}$ ); 27 of the scans were MRIs and 37 were CTs. Interval from stroke to subject examination was $3.51 \pm 4.76 \mathrm{yr}$ (median: $1.75 \mathrm{yr}$, range: $0.42-33.00 \mathrm{yr}$ ). All of the subjects had MCA territory strokes, as determined by the inclusion criteria, but two subjects with grade 5 paresis in addition had infarction in the ACA territory.

Because the first author (V.H.) analyzed all 64 scans, while the second and third authors (S.J. and O.R.) analyzed 16 each, reliability could be assessed for 32 scans. Disagreements were resolved by the senior author (S.N.) without access to data on severity of paresis. There was disagreement on scan angle for two scans; for one of these, the interpretation of the first author (V.H.) was favored, and for the other, that of the second author (S.J.). There was disagreement as to the extent of arm-hand region involvement in three cases. In all three, the measurement of the first author (V.H.) was accepted.

Of all subjects, 96.5 percent had PVWM involvement while 53 percent had minimal $(<25 \%)$ or no ahPCG involvement (Table). Even in subjects with no UL function, 56 percent had minimal infarction of the ahPCG. On the other hand, six subjects with involvement of $>50$ percent of the ahPCG had either a normal motor examination or mild motor impairment, and four subjects with $>50$ percent involvement had moderate impairment in a range that qualified them for CIMT.

The extent of involvement of the posterior PVWM can be seen in the figures. Of the 16 subjects with class 1 to 2 impairment (Figure 1), 7 had some incursion of the deep white matter lesion on the posterior PVWM. Of the 16 subjects with class 3 impairment, the posterior PVWM was spared in 4, but in 2 of these, there was substantial involvement of the cortical arm-hand region (Figure 2). Only two of the subjects with class 4 impairment had sparing of the posterior PVWM, and in one of these, there was major cortical involvement of the armhand region (Figure 3). All of the subjects with class 5

Table.

Number of subjects with damage to precentral gyrus in relation to motor grade.

\begin{tabular}{|c|c|c|c|c|c|}
\hline \multirow{2}{*}{ Motor Grade } & \multicolumn{5}{|c|}{ Damage to Precentral Gyrus } \\
\hline & 0\% & $1 \%-25 \%$ & $26 \%-50 \%$ & $51 \%-75 \%$ & $76 \%-100 \%$ \\
\hline$\overline{1-2}$ & $8(10)$ & $2(61)$ & 0 & $5(68)$ & $1(3,060)$ \\
\hline 3 & $9(349)$ & $2(105)$ & $1(1,290)$ & 0 & $4(2.5)$ \\
\hline 4 & $3(3)$ & $1(90)$ & $1(7)$ & $3(143)$ & $8(1,930)$ \\
\hline 5 & $5(151)$ & $4(237)$ & $2(65)$ & $1(300)$ & $4(1,470)$ \\
\hline Total & $25(159)$ & $9(152)$ & $4(357)$ & $9(119)$ & $17(1,435)$ \\
\hline
\end{tabular}


impairment had extensive involvement of the posterior PVWM (Figure 4). When subjects with some use of the UL (classes 1, 2, and 3) were contrasted with classes 4 and 5 according to presence of posterior PVWM involvement, the difference (19 of 32 with class 1, 2, or 3 impairment; 30 of 32 with class 4 or 5 impairment) was significant $(p=0.002$, Fisher exact test). The correlation of severity of impairment with extent of involvement of the ahPCG was not significant (Spearman $r=0.19, p=0.13$ ).

The extent of involvement of the ahPCG did not appear to be affected by time elapsed between stroke and imaging (Table). The two subjects who did not have PVWM involvement underwent CT scans on the day of the stroke.

\section{DISCUSSION}

In this study of 64 subjects with MCA territory ischemic strokes, 96.5 percent of subjects had deep white matter lesions, most often in the PVWM, while 53 percent had minimal or no ahPCG involvement. This indicates that in half of our subjects, the ahPCG lesion could not account for the paresis, and in the other half, either the ahPCG, the PVWM lesion, or both produced the paresis. There was not a significant correlation between degree of paresis and extent of involvement of the ahPCG. The quality of the images did not allow for a quantitative analysis of the relationship between degree of paresis and extent of involvement of the arm-hand region of the posterior PVWM. However, there was a significant association between presence or absence of a posterior PVWM lesion and functionality of the UL.

Even in subjects with no UL function (class 5), 56 percent had minimal infarction $(<25 \%)$ of the ahPCG. The fact that 10 of 32 subjects with no or only moderate UL motor impairment (grades 1-3 in this study) had involvement of $>50$ percent of the ahPCG provides further support for the concept that extent of ahPCG involvement is an unreliable predictor of arm-hand function. It also suggests that corticospinal and corticobulbar fibers originating in cortical areas other than the ahPCG (e.g., premotor and somatosensory cortex [24]) may suffice to preserve substantial motor function.

Diffusion tensor tractographic (DTT) studies have shown that the corticospinal tract passes through the posterior half of the PVWM, the arm-hand region being centered roughly at the junction of the middle and posterior

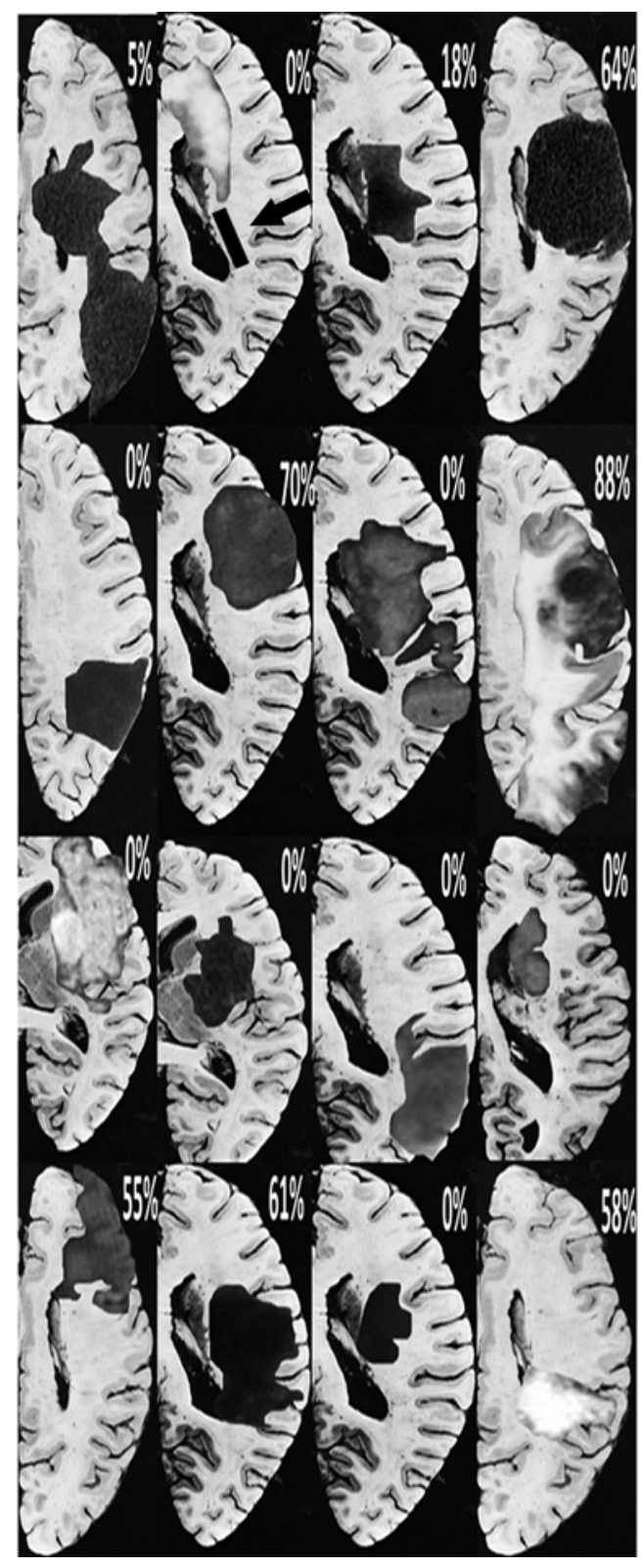

Figure 1.

Axial slice lesion maps in 16 subjects with grade 1 or 2 paresis. Maps of right hemisphere strokes were flipped $180^{\circ}$. The one slice included for each subject is the slice of maximal white matter involvement (taken from Matsui and Hirano [17]). Entire extent of lesion is plotted on each slice, but point of interest is extent of involvement of posterior periventricular white matter, area depicted by rectangle immediately lateral to body of lateral ventricle (first row, second image from left-follow arrow). Armhand region is centered roughly at junction of middle and posterior thirds of this region [25]. Percent involvement of arm-hand portion of precentral gyrus, averaged over all slices, is indicated at top right of each individual subject image. 


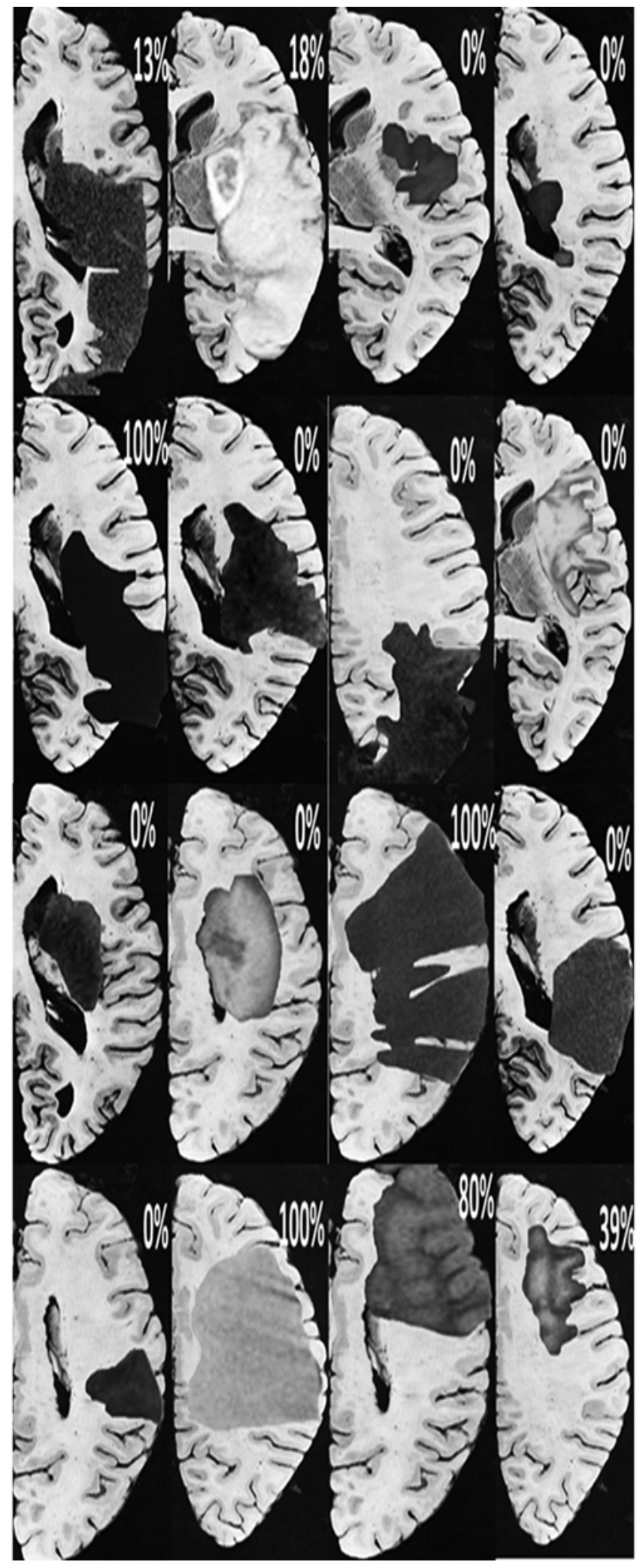

Figure 2.

Axial slice lesion maps in 16 subjects with grade 3 paresis.

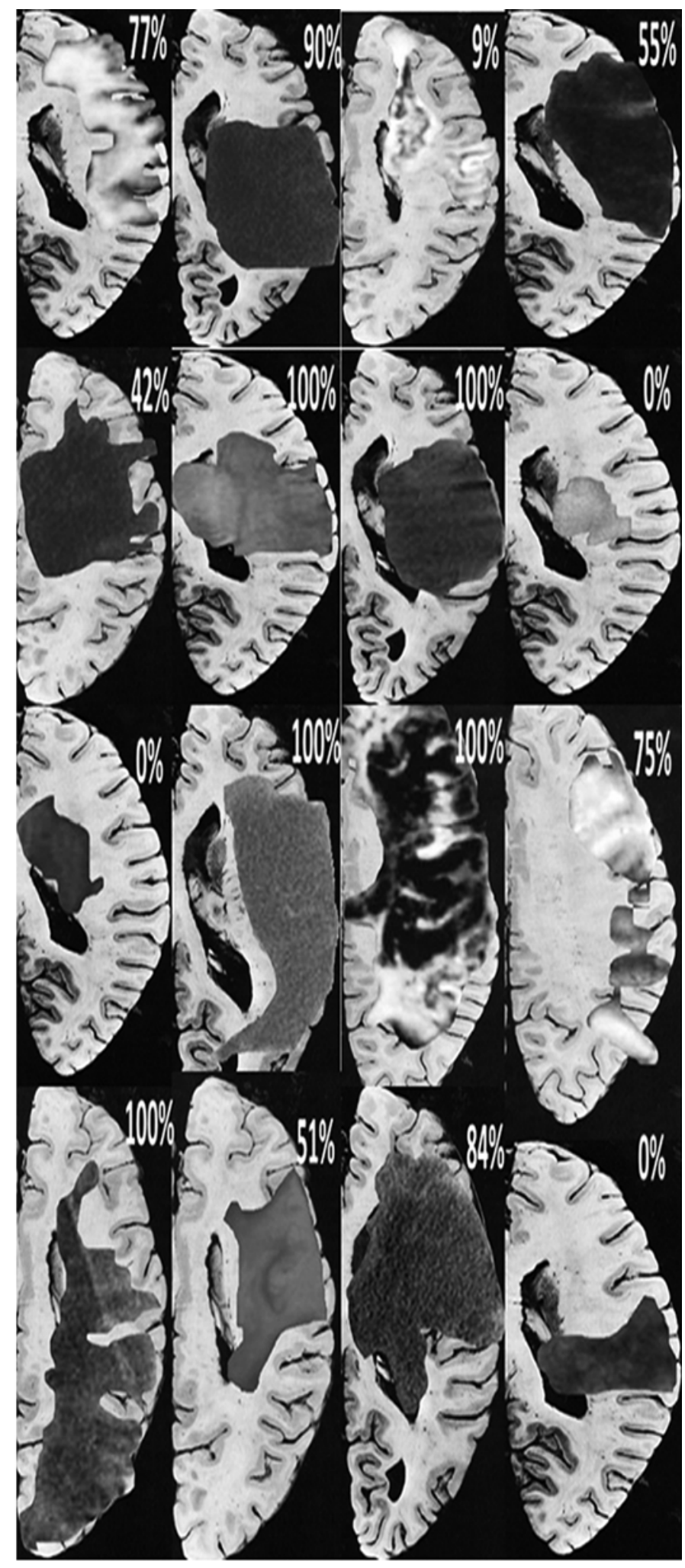

Figure 3.

Axial slice lesion maps in 16 subjects with grade 4 paresis. 


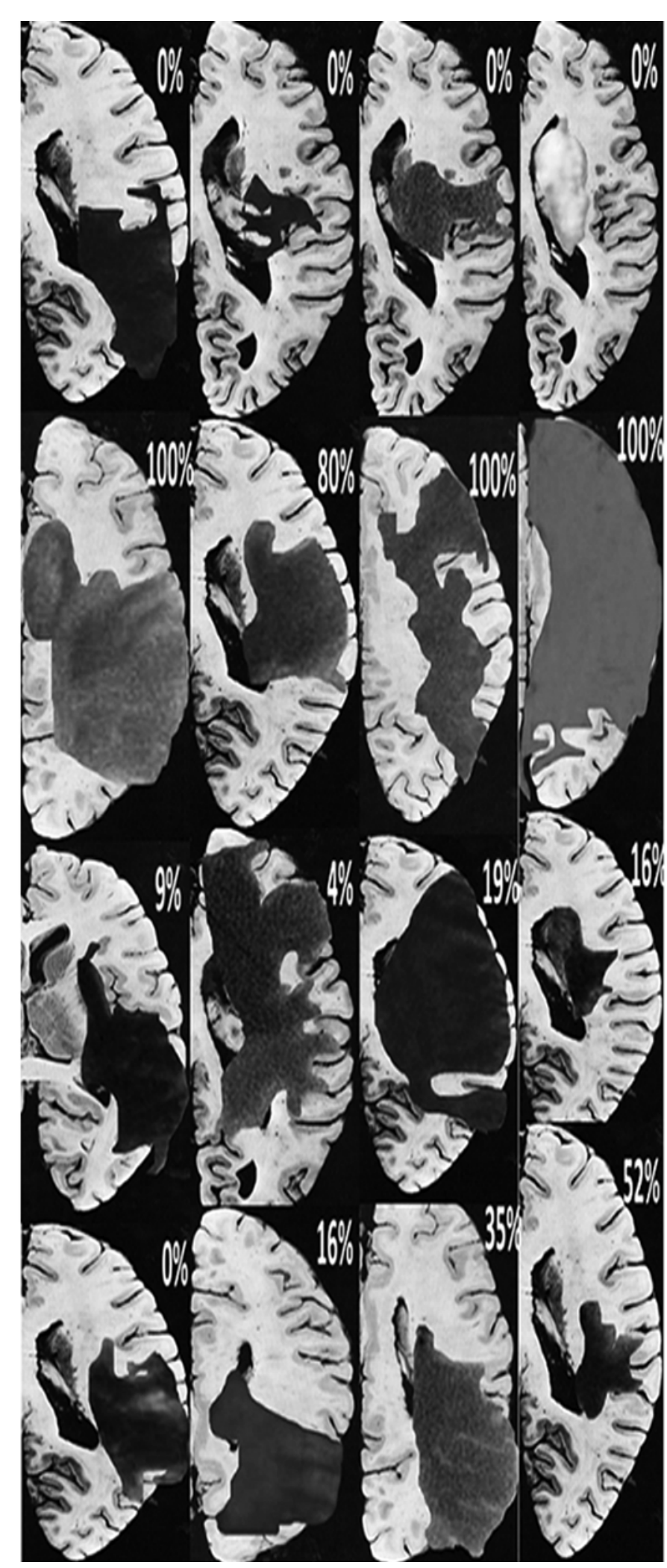

Figure 4.

Axial slice lesion maps in 16 subjects with grade 5 paresis. thirds on scans aligned along the AC-PC line [25]. Stroke studies have shown that lesions in the posterior half of the PVWM are associated with paresis and that the pattern of paresis corresponds with the somatotopic localization defined by DTT studies [1-3]. Our lesion maps are consistent with this localization. More generally, our study is consistent with the results of studies that show that UL motor function after stroke correlates better with axonal integrity in the posterior limb of the internal capsule (as determined by diffusion tensor imaging) than it does with engagement of the ahPCG, as determined by blood oxygen-level dependent fMRI [26].

The modest prevalence of involvement of the ahPCG reflects two factors: (1) even with MCA territory infarcts, the bulk of the lesion is often subcortical, reflecting either occlusion of multiple lenticulostriate vessels by clot in the M1 portion, or infarction of the lenticulostriate endzone (a "desert zone") when clot wedges at the carotid T-junction [27], and (2) even when the cortex is involved, the lesion is characteristically perisylvian and only sometimes extends sufficiently dorsally to involve the ahPCG, reflecting the degree to which posterior cerebral artery-MCA and ACA-MCA end to end anastomoses serve to save cortex from infarction.

These results must be regarded as only suggestive because of the retrospective nature of the radiographic component of this study; the relative insensitivity of the scanning techniques, particularly CT, obtained very soon after stroke; the limitations of the analytic technique applied to the images; and because the potential contribution to paresis of lesions of premotor cortex was not accounted for. It might be noted, however, that the absence of any simple relationship between our results and time between stroke and imaging suggests that our results are not simply an artifact of underdetection of lesions on images obtained early or changes in the imaging appearance of lesions that occur over time. A definitive study would require prospective imaging using stateof-the-art MRI techniques to quantitate damage to the ahPCG, the arm-hand premotor cortex, and the posterior PVWM in relation to the established trajectory of the corticospinal tract.

\section{CONCLUSIONS}

We conclude that the findings of this study provide sufficient support for our hypothesis to warrant prospective studies of the relative contribution of white matter injury 
to paresis in MCA territory stroke employing more refined methodology. The preliminary findings reported here, coupled with results of prior studies, suggest that white matter damage may be a major, if not the principal, determinant of paresis due to hemispheric ischemic stroke, whether large vessel distribution or lacunar, and whether the paresis is UL or lower limb. If supported by further research, these findings have important implications for neurobiological research and for neurobiological and technological strategies for stroke rehabilitation. Further studies of white matter lesions in animals are warranted [28]. Agents that might mitigate white matter damage [29] or agents that might allow axonal regrowth, such as Rho-kinase inhibitors, may be of particular benefit [30]. If the arm-hand region of motor cortex is often spared in MCA stroke, this might provide an opportunity for use of cortically driven neural prostheses in patients with severe paresis, assuming that apparent sparing on structural imaging studies corresponds to substantial sparing at the neuronal level.

\section{ACKNOWLEDGMENTS}

\author{
Author Contributions: \\ Analysis of data: V. S. Hedna, S. Jain, O. Rabbani, S. E. Nadeau. \\ Interpretation of data: V. S. Hedna, S. E. Nadeau. \\ Drafting of manuscript: V. S. Hedna, S. E. Nadeau. \\ Critical revision of manuscript for important intellectual content: \\ V. S. Hedna, S. Jain, O. Rabbani, S. E. Nadeau.
}

Financial Disclosures: The authors have declared that no competing interests exist.

Funding/Support: This material was based on work supported by the VA Rehabilitation Research and Development Service Center of Excellence (grant F2182C).

Additional Contributions: Dr. Nadeau is now with Research Service, Malcom Randall VA Medical Center, Gainesville, Florida. Institutional Review: This study was run under the auspices of the University of Florida Institutional Review Board (IRB-01, protocol 457-99).

Participant Follow-Up: The authors do not plan to inform participants of the publication of this study.

\section{REFERENCES}

1. Jang SH. A review of corticospinal tract location at corona radiata and posterior limb of the internal capsule in human brain. NeuroRehabilitation. 2009;24(3):279-83. [PMID:19458436]

2. Kim JS, Pope A. Somatotopically located motor fibers in corona radiata: Evidence from subcortical small infarcts.
Neurology. 2005;64(8):1438-40. [PMID:15851738]

http://dx.doi.org/10.1212/01.WNL.0000158656.09335.E7

3. Song YM. Somatotopic organization of motor fibers in the corona radiata in monoparetic patients with small subcortical infarct. Stroke. 2007;38(8):2353-55. [PMID:17569873] http://dx.doi.org/10.1161/STROKEAHA.106.480632

4. Cho SH, Kim DG, Kim DS, Kim YH, Lee CH, Jang SH. Motor outcome according to the integrity of the corticospinal tract determined by diffusion tensor tractography in the early stage of corona radiata infarct. Neurosci Lett. 2007; 426(2):123-27. [PMID:17897782]

http://dx.doi.org/10.1016/j.neulet.2007.08.049

5. Konishi J, Yamada K, Kizu O, Ito H, Sugimura K, Yoshikawa K, Nakagawa M, Nishimura T. MR tractography for the evaluation of functional recovery from lenticulostriate infarcts. Neurology. 2005;64(1):108-13.

[PMID:15642912]

http://dx.doi.org/10.1212/01.WNL.0000148477.65273.0C

6. Kunimatsu A, Aoki S, Masutani Y, Abe O, Mori H, Ohtomo K. Three-dimensional white matter tractography by diffusion tensor imaging in ischaemic stroke involving the corticospinal tract. Neuroradiology. 2003;45(8):532-35. [PMID:12856090] http://dx.doi.org/10.1007/s00234-003-0974-4

7. Lie C, Hirsch JG, Rossmanith C, Hennerici MG, Gass A. Clinicotopographical correlation of corticospinal tract stroke: A color-coded diffusion tensor imaging study. Stroke. 2004;35(1):86-92. [PMID:14671237] http://dx.doi.org/10.1161/01.STR.0000106912.09663.EB

8. Zhu LL, Lindenberg R, Alexander MP, Schlaug G. Lesion load of the corticospinal tract predicts motor impairment in chronic stroke. Stroke. 2010;41(5):910-15. [PMID:20378864] http://dx.doi.org/10.1161/STROKEAHA.109.577023

9. Lindenberg R, Renga V, Zhu LL, Betzler F, Alsop D, Schlaug G. Structural integrity of corticospinal motor fibers predicts motor impairment in chronic stroke. Neurology. 2010;74(4):280-87. [PMID:20101033] http://dx.doi.org/10.1212/WNL.0b013e3181ccc6d9

10. Burke E, Cramer SC. Biomarkers and predictors of restorative therapy effects after stroke. Curr Neurol Neurosci Rep. 2013;13(2):329. [PMID:23299824] http://dx.doi.org/10.1007/s11910-012-0329-9

11. Marti-Vilalta JL, Arboix A, Mohr JP. Lacunes. In: Mohr JP, Choi DW, Grotta JC, Weir B, Wolf PA, editors. Stroke: Pathophysiology, diagnosis, and management. 4th ed. New York (NY): Churchill Livingston; 2004. p. 275-99.

12. Duncan PW, Sullivan KJ, Behrman AL, Azen SP, Wu SS, Nadeau SE, Dobkin BH, Rose DK, Tilson JK, Cen S, Hayden SK; LEAPS Investigative Team. Body-weightsupported treadmill rehabilitation after stroke. $\mathrm{N}$ Engl J Med. 2011;364(21):2026-36. [PMID:21612471] http://dx.doi.org/10.1056/NEJMoa1010790 
13. Seitz RJ, Sondermann V, Wittsack HJ, Siebler M. Lesion patterns in successful and failed thrombolysis in middle cerebral artery stroke. Neuroradiology. 2009;51(12):865-71. [PMID:19633834] http://dx.doi.org/10.1007/s00234-009-0576-x

14. Bang OY, Lee PH, Heo KG, Joo US, Yoon SR, Kim SY. Specific DWI lesion patterns predict prognosis after acute ischaemic stroke within the MCA territory. J Neurol Neurosurg Psychiatry. 2005;76(9):1222-28. [PMID:16107355] http://dx.doi.org/10.1136/jnnp.2004.059998

15. Lo AC, Guarino PD, Richards LG, Haselkorn JK, Wittenberg GF, Federman DG, Ringer RJ, Wagner TH, Krebs HI, Volpe BT, Bever CT Jr, Bravata DM, Duncan PW, Corn BH, Maffucci AD, Nadeau SE, Conroy SS, Powell JM, Huang GD, Peduzzi P. Robot-assisted therapy for longterm upper-limb impairment after stroke. N Engl J Med. 2010;362(19):1772-83. [PMID:20400552]

http://dx.doi.org/10.1056/NEJMoa0911341

16. Wolf SL, Winstein CJ, Miller JP, Taub E, Uswatte G, Morris D, Giuliani C, Light KE, Nichols-Larsen D; EXCITE Investigators. Effect of constraint-induced movement therapy on upper extremity function 3 to 9 months after stroke: The EXCITE randomized clinical trial. JAMA. 2006;296(17): 2095-2104. [PMID:17077374] http://dx.doi.org/10.1001/jama.296.17.2095

17. Matsui T, Hirano A. An atlas of the human brain for computerized tomography. New York (NY): Igaku-Shoin; 1978.

18. Damasio H. Human brain anatomy in computerized images. New York (NY): Oxford University Press; 1995.

19. Crafton KR, Mark AN, Cramer SC. Improved understanding of cortical injury by incorporating measures of functional anatomy. Brain. 2003;126(Pt 7):1650-59.

[PMID:12805118]

http://dx.doi.org/10.1093/brain/awg159

20. Meier JD, Aflalo TN, Kastner S, Graziano MS. Complex organization of human primary motor cortex: A highresolution fMRI study. J Neurophysiol. 2008;100(4):18001812. [PMID:18684903] http://dx.doi.org/10.1152/jn.90531.2008

21. Nudo RJ, Milliken GW, Jenkins WM, Merzenich MM. Use-dependent alterations of movement representations in primary motor cortex of adult squirrel monkeys. J Neurosci. 1996;16(2):785-807. [PMID:8551360]

22. Karni A, Meyer G, Rey-Hipolito C, Jezzard P, Adams MM, Turner R, Ungerleider LG. The acquisition of skilled motor performance: Fast and slow experience-driven changes in primary motor cortex. Proc Natl Acad Sci USA. 1998;95(3): 861-68. [PMID:9448252] http://dx.doi.org/10.1073/pnas.95.3.861
23. Weiss KL, Pan H, Storrs J, Strub W, Weiss JL, Jia L, Eldevik OP. Clinical brain MR imaging prescriptions in Talairach space: technologist- and computer-driven methods. AJNR Am J Neuroradiol. 2003;24(5):922-29. [PMID:12748095]

24. Stinear CM, Barber PA, Smale PR, Coxon JP, Fleming MK, Byblow WD. Functional potential in chronic stroke patients depends on corticospinal tract integrity. Brain. 2007;130(Pt 1):170-80. [PMID:17148468]

http://dx.doi.org/10.1093/brain/awl333

25. Han BS, Hong JH, Hong C, Yeo SS, Lee D, Cho HK, Jang $\mathrm{SH}$. Location of the corticospinal tract at the corona radiata in human brain. Brain Res. 2010;1326:75-80.

[PMID:20219443]

http://dx.doi.org/10.1016/j.brainres.2010.02.050

26. Qiu M, Darling WG, Morecraft RJ, Ni CC, Rajendra J, Butler AJ. White matter integrity is a stronger predictor of motor function than BOLD response in patients with stroke. Neurorehabil Neural Repair. 2011;25(3):275-84. [PMID:21357529] http://dx.doi.org/10.1177/1545968310389183

27. Nadeau SE, Crosson B. Subcortical aphasia. Brain Lang. 1997;58(3):355-402, discussion 418-23. [PMID:9222518]

28. Sozmen EG, Hinman JD, Carmichael ST. Models that matter: White matter stroke models. Neurotherapeutics. 2012;9(2): 349-58. [PMID:22362423] http://dx.doi.org/10.1007/s13311-012-0106-0

29. Liu XS, Chopp M, Kassis H, Jia LF, Hozeska-Solgot A, Zhang RL, Chen C, Cui YS, Zhang ZG. Valproic acid increases white matter repair and neurogenesis after stroke. Neuroscience. 2012;220:313-21. [PMID:22704966] http://dx.doi.org/10.1016/j.neuroscience.2012.06.012

30. Walmsley AR, Mir AK. Targeting the Nogo-A signalling pathway to promote recovery following acute CNS injury. Curr Pharm Des. 2007;13(24):2470-84. [PMID:17692015] http://dx.doi.org/10.2174/138161207781368611

Submitted for publication October 29, 2012. Accepted in revised form February 27, 2013.

This article and any supplementary material should be cited as follows:

Hedna VS, Jain S, Rabbani O, Nadeau SE. Mechanisms of arm paresis in middle cerebral artery distribution stroke: Pilot study. J Rehabil Res Dev. 2013;50(8):1113-22. http://dx.doi.org/10.1682/JRRD.2012.10.0194

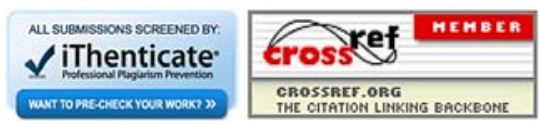


